\title{
Abnormal Maritime Activity Detection in Satellite Image Sequences Using Trajectory Features
}

\author{
Chin-Shyurng Fahn, Jer Ling, Ming-Yuan Yeh, Po-Yen Huang, and Meng-Luen Wu
}

\begin{abstract}
Maritime security has gained much attention these days because of the frequent interaction between countries with marine borders. Surveillance systems on land are installed on a fixed point, while there is no mounting point for marine surveillance. Fortunately, with the advancement of aerospace technology, the maritime monitoring is possible with continuous satellite image sequences. In this paper, we proposed a complete scheme for maritime surveillance using image sequences on embedded satellite systems, including maritime object detection, tracking, and abnormal activity classification. The recall rate for object detection is $78 \%$. The recall rate for tracking is $91 \%$. The recall rate for anomaly detection is $78 \%$. The FPS is one frame per second on a low power-consuming device installable on a satellite system. The proposed scheme is helpful for the national defense and is labor saving for coast guard affairs.
\end{abstract}

Index Terms-Satellite image analysis, intelligent surveillance, ship detection, abnormal detection, trajectory analysis.

\section{INTRODUCTION}

Satellite images are the images from far above the globe, and captured by high-end cameras with high resolution. These images were only obtainable by governments or military units. Nowadays, because of the advancements in aerospace technology, private companies or institutions are able to receive and provide these images for commercial use. With the improvement of image capturing speed, the satellite images are possible for surveillance use. In this paper, we focus on the surveillance on maritime activities using satellite image sequences.

The goal of this paper is to find abnormal activities in maritime objects, which is a type of intelligent surveillance. Generally, intelligent surveillance systems work on real-time surveillance video. However, most satellites work on solar powered battery that the embedded processors are power saving in order to extend the battery life. As a result, the frame rate of images captured by satellites is limited. Therefore, the satellite image sequence is used instead of

Manuscript received October 9, 2018; revised February 10, 2019. This work was supported in part by the National Space Organization (NSPO), Hsinchu, Taiwan under Grant NSPO-S-106150.

Chin-Shyurng Fahn is with Dept. of Computer Science and Information Engineering, National Taiwan University of Science and Technology, Taipei 10607, Taiwan (e-mail: csfahn@mail.ntust.edu.tw).

Jer Ling, Ming-Yuan Yeh, and Po-Yen Huang are with Dept. of System Engineering, National Space Organization, Hsinchu 30078, Taiwan (e-mail: se01@nspo.narl.org.tw, marco@nspo.narl.org.tw).

Meng-Luen Wu is with Dept. of Computer Science and Information Engineering, National Taiwan University of Science and Technology, Taipei 10607, Taiwan (e-mail: d10015015@mail.ntust.edu.tw). real-time surveillance video. Fortunately, the satellites are flying far above the maritime activities, and the ship movements take much time in the image sequence, the object trajectory can be analyzed effectively.

The daytime and night time satellite image are quite different. In the night time image, only lights are observable. Therefore, we choose the images from day time only. On the other hand, most maritime activities occur between the continent and the sea, we select the satellite image sequences in these areas as the region of interest. We show some target satellite images in Fig. 1, which includes seashores, rivers, and islands that maritime activities often exist.

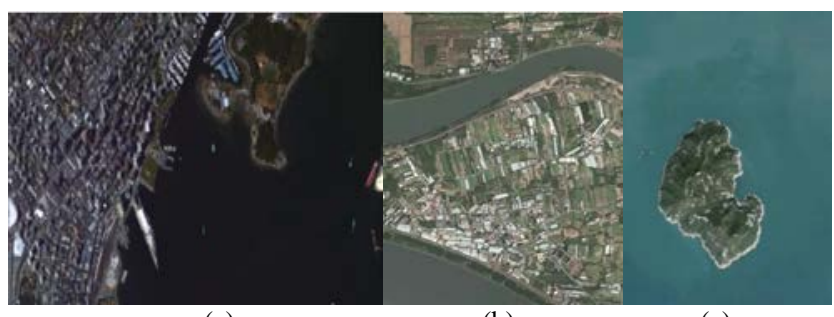

(a)

(b)

(c)

Fig. 1. Satellite images of interest: (a) seashore; (b) river; (c) island.

The interaction between countries is increasing, including international trade, commerce, and tourist activities, which provides the economic grows between countries. However, some problem also arises. There are various kinds of abnormal maritime activates, such as smuggling, piracy, stowaway, trespassing of national boundaries, and so on. The continual abnormal maritime activity is not only a legal issue but also a national defense concern. From statistics, the number of suspicious maritime activity is increasing, which forces government to invest more resources to deal with the problem. To reduce the personnel costs on this kind of affair, government seek for intelligent surveillance systems as a solution.

Unlike traditional surveillance systems which depend on stationary surveillance cameras, some abnormal maritime activities occur in the middle of the sea, which means no installation point is available for the camera. Satellite images with high resolution image capture capabilities is an ideal alternative in this regard.

In this paper, we propose a novel surveillance system to detect the abnormal maritime activities in day time. There are several parts in the system. First, we use image pre-processing techniques to mark all possible objects in an image. In all of the possible objects, the machine learning method is applied to filter out the interested ones, primarily ships. Second, once an object has been detected, the system keeps track on it in order to formulate its moving trajectory. The normal and abnormal trajectories are both recorded and 
sent into another machine learning model, which is used to detect abnormal activities. Once trained, the machine learning model is able to classify whether a moving object in the maritime activity is abnormal. Fig. 2 shows the flowchart of our proposed method.

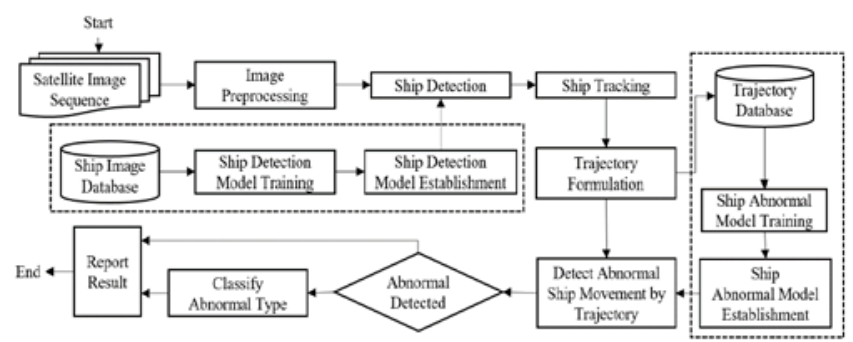

Fig. 2. Flowchart of the abnormal maritime activity detection.

\section{RELATED WORK}

With the advancement of aerospace and satellite technology, in 1997, Lin et al. [1] used the European remote sensing (ERS) and Synthetic-aperture radar image from the satellite for ship wake detection to monitor the ship traffic. In 2005, the SAR image is applied for maritime surveillance [2] for the use of automatic identification system (AIS) to prevent collisions among maritime vehicles. Sandirasegaram et al. [3] proposed to validate the SAR image for better ship detection accuracy. Above authors chose SAR image the most because of the inferior quality of RGB image in cameras The disadvantage of SAR image is that the detail of the anomaly cannot be comprehend by humans easily. Currently, part of the satellites is equipped with high resolution cameras, and satellite surveillance using cameras is possible. In 2017, Zhang et al. [4] proposed a moving object detection method on satellite RGB image. In our methods, in addition to object detection, we further propose methods for object tracking and abnormal behavior detection on these objects.

\section{Extraction Of Moving ObJects in Maritime ACTIVITY}

In this section, we propose the method of the extraction of moving objects in maritime activity. There are two parts in the extraction, including detection and tracking. To detect the objects, morphological operation is used to mark up all possible objects, and machine learning method is applied to filter and remove the false positive detections. To track the moving objects, the cam-shift method is utilized to trace the movement of objects.

\section{A. Moving Objects Detection}

The objects in maritime activities are surrounded by sea water, and appeared as contours in an image. The contours are possible moving objects in the maritime activity, as seen in Fig. 3(a). To find the contours, firstly, convert the input frame into a binary image. The image is converted into greyscale image as Fig. 3(b) shows, and set a threshold [5] slightly above the average sea intensity. Pixels above the threshold are set as foreground and the others are set as background, and the foreground mask is produced as Fig. 3(c) shows. To reduce unwanted noise and fill up unexpected holes in the mask, the morphological operation is performed as a solution to produce a refined mask. Empirically, applying one-unit dilation operations followed by two-unit erosion operations gives the optimal result, as in Fig. 3(d) shows. Subsequently, find the connected components [6] in the refined mask, and mark each of the component as different objects. In Fig. 3(e), we see some unwanted regions are also selected as objects, and we can ignore them by removing regions with illogical contour area and aspect ratios. Fig. 3(f) shows the result after removing.
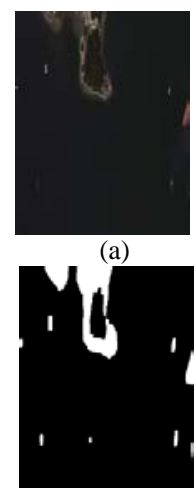

(d)

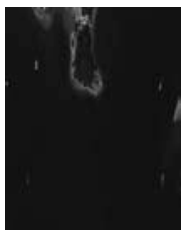

(b)

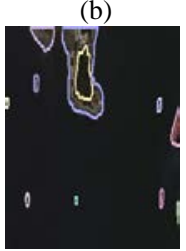

(e)

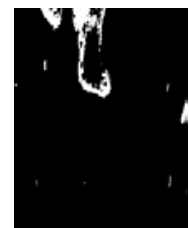

(c)

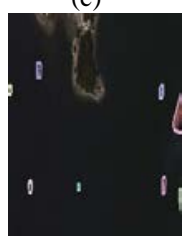

(f)
Fig. 3. The process of detection of object contours: (a) original image; (b) grayscale image; (c) binarization; (d) morphological dilation; (e) find contours in binary image; (f) filtering of unnecessary contours.

After the above process, the possible moving objects are extracted logically. By using this method, most of the unwanted contours are eliminated, and much computational time is saved. However, some extracted contours do not include maritime objects, and should be removed by other steps.

The machine learning method as a filter to select objects. In the machine learning process, the training samples are chosen from image contents in the extracted contour, and are labelled as two types, objects and non-objects. We use support vector machine (SVM) [6] to build a binary classifier model, and calculate the center of gravity and aspect ratio as inputs. After the model is built, we input every extracted contour to the model to verify whether it is an object in maritime activity. As a result, the desired objects in the activity are obtained.

\section{B. Moving Objects Tracking}

To trace the moving activity of the extracted objects, the tracking techniques are applied on these detected objects. The cam-shift method [7], which is an improvement of mean-shift [8], is used as the algorithm to track maritime objects in our method. The flow chart is shown in Fig. 4. Most of the cam-shift algorithm is based on mean-shift. In mean-shift, the user or system firstly select an interested region, and tracking is performed in subsequent frames. The images are converted to HSV color space and produce the histograms of these channels. The main working principal of mean-shift is to compute the likelihood as of interested regions of two adjacent frames as a 2-D probability density function [9]. In each frame, calculate the mass center of the probability density function, and adjust the tracking window to the center to the mass iteratively until converge. Different from mean-shift tracks objects with the fixed size, the 
cam-shift adjusts the tracking size adaptively. The cam-shift is a consecutive mean-shift, which adjusts the size of the mean-shift iteratively until converge.

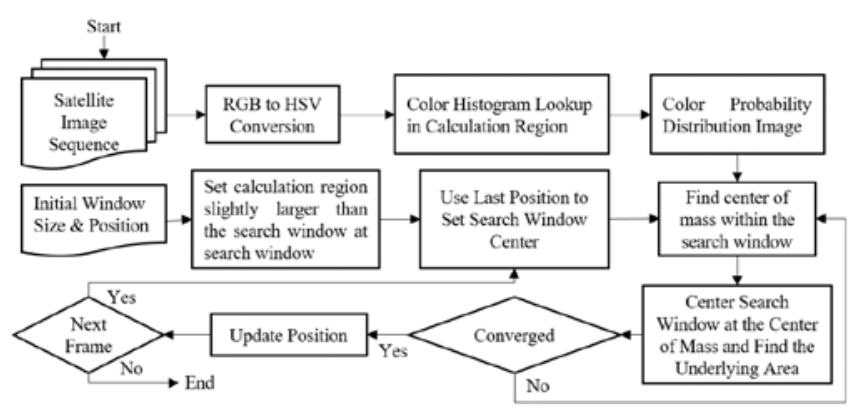

Fig. 4. Flowchart of the cam-shift tracking method.

\section{ABnormal Behavior Detection}

In the previous section, the trajectory of each maritime object is obtained by tracking of their movement. The abnormal behavior detection is possible by analyzing the trajectory. The angle of the centroid position and moving direction is an important feature for detection. By categorizing the angles, there are four major abnormal behaviors, namely "stop," “wandering," “swapping,” and "gathering." We describe the detection of four types of abnormal behaviors in this section.

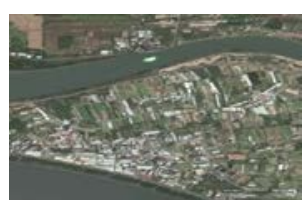

(a)

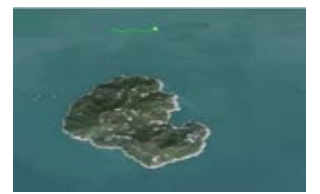

(c)

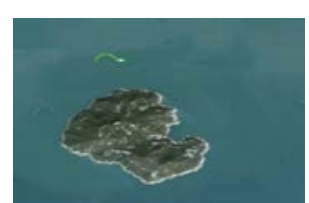

(b)

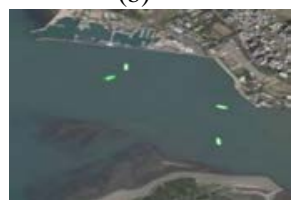

(d)
Fig. 5. Four types of abnormal behaviors: (a) stop; (b) wandering; (c) swapping; (d) gathering.

There are four types of abnormal behaviors to be detected, and these behaviors are illustrated in Fig. 5. The "stop" behavior means the object stops at a point in the middle of the sea for a period of time, which may because there is some damage on the object that it stops moving. The "wandering" behavior indicates an object moving around a region, which can be scouting, patrolling, or investigating some targets. The "swapping" behavior means the object is zigzagging in its path, which often occurs when something is escaping from pursuit. The "gathering" means the objects are close to each other, which may because of piracy, smuggling, or illegal trading. These four types of abnormal behaviors are most observed anomalies.

\section{A. Detection of Abnormal Behaviors}

The trajectory is analyzed for abnormal behavior detection. The trajectory is composed of a series of coordinates of a moving object, and the time of each coordinate is recorded as a reference. For the four abnormal behaviors, we design four detectors for each type of anomaly respectively. For the "stop" behavior detector, if the coordinates in the trajectory of an object keep static for a period of time, empirically 30 minutes, the object is detected as "stop."

For the wandering behavior, the average rotation degree is used as a detector. Because of the characteristic of moving objects in the water, objects cannot make turns with high degree of angle at a time, if the object moves around the region, it must have high degree of angle in most of the moving steps. Fig. 6 depicted the calculation method of rotation degree at certain point. To get the rotation degree of point $\mathrm{B}$, choose other two points, $\mathrm{A}$ and $\mathrm{C}$ with the same distance to $\mathrm{B}$ from both sides of the trajectory, the angle degree of the intersection is calculated as the rotation degree of the point. If the average degree of the trajectory of a moving object is higher than twice the average of all trajectories, it is detected as abnormal.

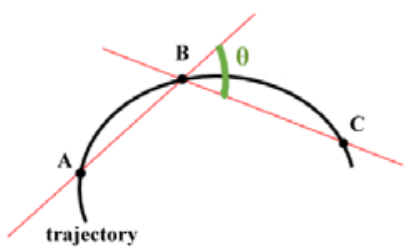

Fig. 6. Rotation degree calculation for wandering behavior detection.

For the swapping detection, similarly, calculate the angle degree as in wandering detection does. Based on the wandering detection method, the swapping detection records the orientation from the angle degree. The direction is obtained from the difference from the current orientation and the orientation in the frame ten minutes before. If the difference keeps changing back and forth between negative and positive, the behavior is detected as swapping.

For the gathering detection, calculate the distance between any two objects, and set two thresholds, which are time and distance. If the distance between any two objects is below the distance threshold and keeps the state for a period above the time threshold, the objects are detected as gathering.

So far, the detection method of the four abnormal behaviors are explained. To improve the accuracy and performance of the overall abnormal detection, we use the decision tree C5.0 method as a machine training method to generate a decision tree using the binary output of the four detectors as features [10], which the result is as Fig. 7 shows.

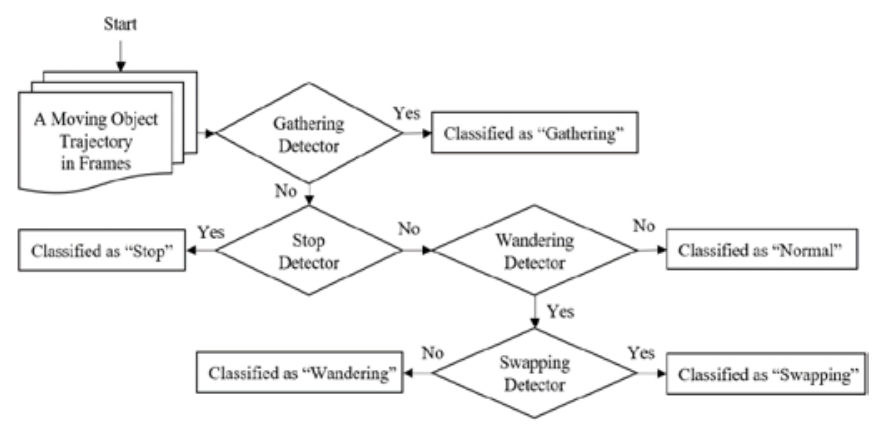

Fig. 7. Classification rule of abnormal events of maritime activities.

The tree-structured classifier is used of four parallel independent detectors. In the tree, firstly detect whether the moving objects are gathering. Subsequently, detect whether they are in the static state in a period of time. The wandering 
and swapping are two similar behaviors, but according to the decision tree generation, detect wandering prior to swapping gives the better performance and accuracy. A moving object is classified as normal if not gathering, stopping, and wandering.

\section{EXPERIMENTAL RESULTS AND DISCUSSION}

In this section, we show experimental result of each process of the abnormal maritime activity detection, including object detection, tracking, and abnormal activity detection. The proposed method is workable on power-consuming ARM based embedded systems, such as Raspberry Pi. We will first describe the experimental setup and list the accuracy of experimental result.

\section{A. Experimental Setup}

The satellite image is of high resolution; the resolution of each image frame is $1,920 \times 1,080$. We use the satellite image sequence as the experimental input. The minimum size of the maritime objects is 100 pixels, and 2,500 pixels the largest. In the object detection part, 326 object training samples and 1,152 non-object training samples are used. Our proposed system is also run on a low power consumption ARM based system, which Raspberry Pi 3 is chosen as a solution, which includes Broadcom BCM 2837 SoC, 4-core ARM Cortex-A35 1.2GHz CPU, Broadcom VideoCore IV GPU, and with 1GB LPDDR2 900MHz main memory.

\section{B. Object Detection Results}

In the ship detection, the system selects objects within all extracted contours, and filter them with our trained SVM model. There are two types of incorrect detections, which are false positive (non-object detected as an object) and false negative (object not detected). Figure 8 shows the detected objects in green and blue bounding box. The blue bounding box represents the object captured based on the dilated binary mask, and the green one is the actual object region obtained by shrinking the dilated area.

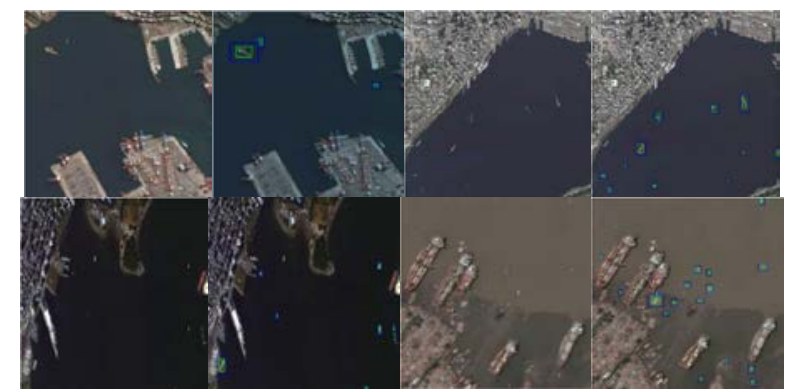

Fig. 8. Object detection result: original images on the left, and detected results on the right.

The confusion matrix for the detection accuracy is shown in Table 1. We evaluate our detector under satellite images with high environmental complexity. Using recall rate to calculate accuracy, and the result is approximately $78 \%$.

TABLE I: CONFUSION MATRIX OF OBJECT DETECTION

\begin{tabular}{lll}
\hline Actual & Detected & Non-object \\
\hline Object & 35 & 10 \\
Non-object & 10 & N/A \\
\hline \hline
\end{tabular}

\section{Object Tracking Results}

To evaluate the effectiveness of object tracking, we define a success tracking in the case that if an object has been tracked successfully from entering to leaving the field of view of the image sequence; otherwise, the object is missed. We track the objects in four video sequences, and list the number of successfully tracked and missed objects in Table 2. The success rate of tracking is approximately $91 \%$.

TABLE II: RESULT OF MARITIME OBJECT TRACKING

\begin{tabular}{|c|c|c|c|c|}
\hline Result & No. 1 & No. 2 & No. 3 & No. 4 \\
\hline Success Tracking & 3 & 3 & 1 & 3 \\
\hline Missed & 0 & 0 & 0 & 1 \\
\hline
\end{tabular}

\section{Abnormal Detection Results}

Our abnormal detector can detect whether a moving object is abnormal, and is able to classify four types of anomalies if detected as abnormal. In the first part, we only examine whether a moving object is normal, in the second part, we further classify the type of anomaly if detected as abnormal. Some results of the first part of abnormal detection are shown in Table 3.

TABLE III: CONFUSION MATRIX OF ABNORMAL DETECTION

\begin{tabular}{lcc}
\hline Detected & Normal & Abnormal \\
\hline Normal & 3 & 0 \\
Abnormal & 0 & 8 \\
\hline \hline
\end{tabular}

From Table 4, we can see most normal objects are detected as normal, and only two abnormal behaviors are detected as normal. When using recall rate $\frac{T P}{T P+F N}$ as a measure of accuracy, recall rate is $78 \%$.

TABLE IV: ACCURACY OF SHIP ABNORMAL DETECTION

\begin{tabular}{ccc}
\hline Detected & Normal & Abnormal \\
OBJECT & 7 & 2 \\
Non-OBJECT & N/A & N/A \\
\hline \hline
\end{tabular}

TABLE V: CONFUSION MATRIX OF FOUR TYPES OF ANOMALIES

\begin{tabular}{|c|c|c|}
\hline Actual & Stop & Other \\
\hline Stop & 1 & 0 \\
\hline Other & 0 & 0 \\
\hline Actual & Gather & Other \\
\hline Gather & 1 & 0 \\
\hline Other & 0 & 0 \\
\hline Actual & Swap & Other \\
\hline Swap & 1 & 0 \\
\hline Other & 0 & 0 \\
\hline Actual & Wander & Other \\
\hline Wander & 1 & 0 \\
\hline Other & 0 & 0 \\
\hline
\end{tabular}

The proposed method also classifies the four types of anomalies correctly. In Table 5, we examined four trajectories, and each of them are classified into their 
respective types of anomalies, which are wandering, swapping, stopping, and gathering respectively. The performance of the system is capable of detecting the anomalies on the satellite system. The detection speed can reach one frame per second on a low power consuming Raspberry Pi 3 platform. Therefore, the pro-posed scheme is workable on the maritime abnormal activity detection on the satellite system

\section{CONCLUSION}

The abnormal maritime activity has been paid attention of these days because of the frequent interaction between countries. Automatic surveillance and alarm is helpful for the national defense and is labor saving. In this paper, we proposed a complete scheme for abnormal maritime activity detection. We proposed a series of methods, including object detection, tracking, and abnormal behavior detection and classification for maritime activities on satellite image sequences in embedded systems. The recall rate for object detection is $78 \%$. The recall rate for tracking is $91 \%$. The recall rate for anomaly detection is $78 \%$. The FPS is one frame per second on a low power-consuming device installable on a satellite system. Because of the processing power is limited on embedded devices on satellites, the accuracy for some of the part is limited. In the future, we would also employ additional imaging devices and obtain more information to retrieve more clues in abnormal maritime behaviors.

\section{ACKNOWLEDGMENT}

The authors thank Ying-Tse Lee, Chi-Leong Lok, Yi-Fang Lee, Yi-Lun Wang, Zi-Yu Chen, and Tzu-Yuan Wu who are the members of the research team affiliated to National Taiwan University of Science and Technology (NTUST) for enthusiastically participating the development of the abnormal maritime activity detection system.

\section{REFERENCES}

[1] I. Lin, L. K. Kwoh, Y. C. Lin, and V. Khoo, "Ship and ship wake detection in the ERS SAR imagery using computer-based algorithm," in Proc. of the IEEE International Geoscience and Remote Seneing Symposium, 1997, pp. 151-153.

[2] T. Wahl, G. K. Høye, A. Lyngvi, and B. T. Narheim, "New possible roles of small satellites in maritime surveillance," Acta Astronautica, vol. 56, no. 2, pp. 273-277, 2005.

[3] N. Sandirasegaram and P. W. Vachon, "Validating targets detected by SAR ship detection engines," Canadian Journal of Remote Sensing, vol. 43, no. 5, pp. 451-454, 2017.

[4] X. Zhang and J. Xiang, "Moving object detection in video satellite image based on deep learning," in Proc. of the LIDAR Imaging Detection and Target Recognition Conference, 2017, pp. 106054H-1-106054H-8.

[5] R. C. Gonzalez and R. E. Woods, Digital Image Processing, 4th Ed. New York: Pearson, 2018, pp. 809-813.

[6] C. Cortes and V. Vapnik, "Support-vector networks," Machine Learning, vol. 20, no. 3, pp. 273-297, 1995.

[7] S. Suzuki, "Topological structural analysis of digitized binary images by border following," Computer Vision, Graphics, and Image Processing, vol. 30, no. 1, pp. 32-46, 1985.

[8] Y. Cheng, "Mean shift, mode seeking, and clustering," IEEE Transactions on Pattern Analysis and Machine Intelligence, vol. 17, no. 1, pp. 790-799, 1995.
[9] G. R. Bradski, "Real time face and object tracking as a component of a perceptual user interface," in Proc. of the IEEE Workshop on Applications of Computer Vision, 1998, pp. 214-219.

[10] S. M. Liu, "On SVM decision-based human activity recognition techniques for single camera video,” M. S. Thesis, Department of Computer Science and Information Engineering, National Taiwan University of Science and Technology, Taipei, Taiwan, 2011.

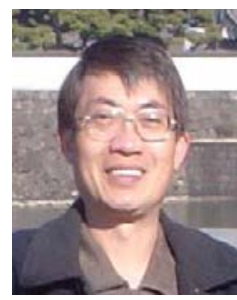

Chin-Shyurng Fahn received the Ph.D. degree in electrical engineering from National Cheng Kung University, Tainan, Taiwan in 1989. From August 1991 to July 2002, he served as an associate professor in the Department of Electrical Engineering at National Taiwan University of Science and Technology (NTUST), Taipei, Taiwan. Since August 2002, he has been on the Department of Computer Science and Information Engineering of the same university, and promoted to be a full professor in 2015. His current fields of interest include image processing, pattern recognition, robot vision, video surveillance, computational aesthetics, and machine learning. His biography is also listed in the Marquis Who's Who in the World and Who's Who in Science and Engineering.

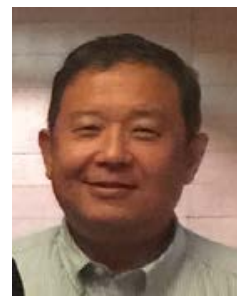

Jer Ling received the Ph.D. degree from the Department of Aerospace Engineering, the University of Michigan, Ann Arbor, USA in 1992. Since then, he joined the National Space Organization (NSPO), Taiwan. His major specialties and research interests are with the satellite system engineering, sensors and the remote sensing instrument, and solar UAV. He has participated almost all programs of NSPO, Formosat-1, Formosat-2 and Formosat-5. Since 2005, he has been the Division Director of System Engineering, and has also worked on developing the solar high altitude UAV. Currently, he leads the development work for the very-high resolution remote sensing satellite, TDI CMOS imaging sensor, and AI on the remote sensing.

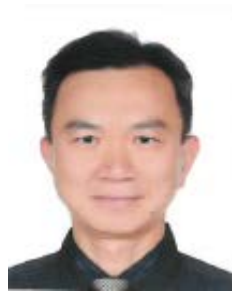

Ming-Yuan Yeh received the B. S. degree from the Department of Mechanical Engineering in National Taiwan University, Taipei, Taiwan in 1984. He joined the NSPO, Taiwan in 1994. His major specialties and research interests are with the satellite system engineering, sensors and the remote sensing instrument, and solar UAV. Currently, he is the researcher of System Engineering Division of NSPO.

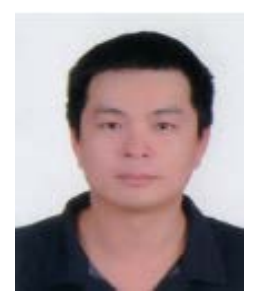

Po-Yen Huang received the B. S. degree from the Department of Electrical Engineering in National Central University, Taoyuan, Taiwan in 2007. He joined the NSPO, Taiwan in 2013. His majo specialties and research interests are with the satellite system engineering, sensors and the remote sensing instrument. Currently, he is the assistant engineer of System Engineering Division of NSPO.

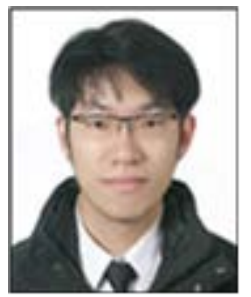

Meng-Luen Wu received the B. S. degree in law and the B.S. degree in computer science and information engineering from National Taipei University, Taipei, Taiwan in 2011, and the Ph.D. degree in computer science and information engineering from NTUST, Taipei, Taiwan in 2017. He is currently the postdoctoral researcher in NTUST. From 2012 to 2013, he was a research assistant with the Institute of Information Science, Academia Sinica, Taipei, Taiwan. His research interest includes image processing, computer vision, artificial intelligence, and data mining. 